\title{
Image Processing by using Discrete Laguerre Wavelets Transform (DLWT)
}

\author{
Bavanari Satyanarayana \\ Department of Mathematics \\ Acharya Nagarjuna University \\ Andhra Pradesh, India
}

\author{
Asma Abdulelah \\ Abdulrahman \\ Department of Mathematics \\ Acharya Nagarjuna University \\ Andhra Pradesh, India
}

\author{
Y. Pragathi Kumar \\ Department of Mathematics \\ College of Natural and \\ Computational Sciences \\ Adigrat University, Adigrat, Ethiopia
}

\begin{abstract}
In this paper, the author used Discrete Laguerre Wavelets Transform (DLWT) and also analyzed to reach the extraction of the resolution tree from Discrete Laguerre Wavelets Transform's Coefficients (DLWT) ${ }_{\mathrm{C}}$ ' which resulted in the extraction of filters that will help to derived the new laws and algorithms and it makes the new wavelet to take its role in the image processing. Some examples and algorithms also included in this paper.
\end{abstract}

\section{General Terms}

Discrete Laguerre Wavelets Transform

\section{Keywords}

Discrete Laguerre Wavelets Transform (DLWT), Inverse Discrete Laguerre Wavelets Transform (IDLWT), Low pass filter, High pass filter, Decomposition, Reconstruct, Processed images, Compressed images.

\section{INTRODUCTION}

Over the years, the conversion of waves has been considered as a mathematical way of dealing with signals, which in turn changes from time domain to frequency domain [1,2]. In addition, it provides more accurate information for wave data in other signal analysis techniques [7].

Wavelets tool box have the advantage of processing and analysis (signal, images, and data) that show regular behavior with sudden changes. So algorithms and probabilities analyze discrete wavelets transform (DWT), decimated and non decimated wave packets, double tree and wave forms [13].

All because of their containment tools for continuous wavelets transforms (CWT) algorithms [8]. Which will be explained in this paper. Wavelet transform has been used as an such as audio, compression and computer graphics [3-5]. And because of its own self a similarity in its ability to above applications making it one of the modern advances to Fourier transform [11]. The wavelet transform technique in analyzing several types of signals such as radar and earth quake signals is best for their ability to analyze unstable phenomenon [12].

The wavelet transform its representation form because of a wide range of process and functions that done so it has become the standard tool in signal and de noising [6]. This work was done by working on a soft ware system to sharpen the edges and will applied DLWT for image processing by using wavelets and its filters matrix and explained how programming it by using MATLAB program and VISUAL BASIC program.

\section{WAVELET TRANSFORMATION}

Wavelet transformation $[9,10]$ is a variable analysis in terms of division of the frequency window over time, giving it a force in analysis compared to Fourier transform, as shown in Fig.1, and conversion is one of the most important modern transformations used by researchers in many applications because it contains features and characteristics based on statistical speculation that play a large role in digital processing. It also has the advantage of being reference or analyzing images from multiple levels of details and this is one of the most important features used to enhance the edges of digital images.

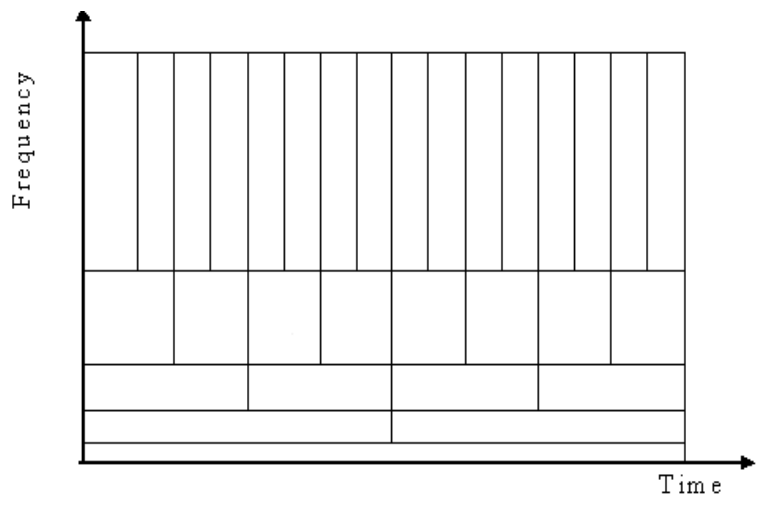

Fig .1 The waveguide representation (frequency-to-time division)

\section{FAST DISCRETE LAGUERRE WAVELETS TRANSFORM FDLWT ALGORITHM}

This section describes the following topics.

\subsection{To Calculate DLWT and IDLWT \\ by using Filters}

In framework of multi resolution of an orthogonal wavelet, this section begins with the scaling function $\vartheta$ defined by

$$
\left\{\vartheta_{n, m}(t)\right\}_{n, m \in z}=2^{-n / 2} \vartheta\left(2^{-n} t-m\right) \quad \forall n, m \in Z
$$

and Discrete Laguerre Wavelets Transform $\rho$

$$
\rho_{n, m}(t)=2^{-n l 2} \rho\left(2^{-n} t-m\right), \forall n, m \in Z
$$

Essential relations are the twin-scale relations

i.e., expansion equation or perfect equation

$$
\frac{1}{2} \vartheta\left(\frac{t}{2}\right)=\sum a_{m} \vartheta(x-m)
$$


The filters contributory in DLWT and in IDLWT and carefully attached $\left(a_{m}\right), m \in Z$. Let $\vartheta$ and $\rho$ have tight backing, it shows that these filters or series contain a limited number of non-zero elements what follows is the low filter for this filter and denotes this filter W. From the above low frequencies are available for their preservation the high ones. It has a Finite Installment Response (FIR) of length $2 \mathrm{M}$, its sum is 1 and its norm is $\frac{1}{\sqrt{2}}$. Four filters can be defined by using filter's properties as follows.

step 1:- The filter $\mathrm{W}$ is used.

step 2:- With finite installment response.

Step 3:- The decomposition filters are noted specified by the final $\mathrm{D}: \mathrm{L}_{0} \mathrm{D}, \mathrm{H}_{\mathrm{i}} \mathrm{D}$

Step 4:- The initial $\mathrm{L}_{0}$ is first low-pass.

Step 5:- The initial $\mathrm{H}_{\mathrm{i}}$ is second is high-pass.

Step 6:- The final $\mathrm{R}$ has two reconstruction filters.

The above steps explain four filters in the following figures.

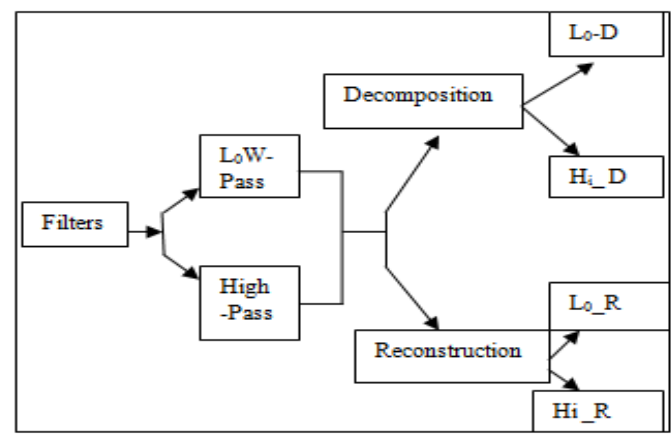

Fig.2 (Four filters)

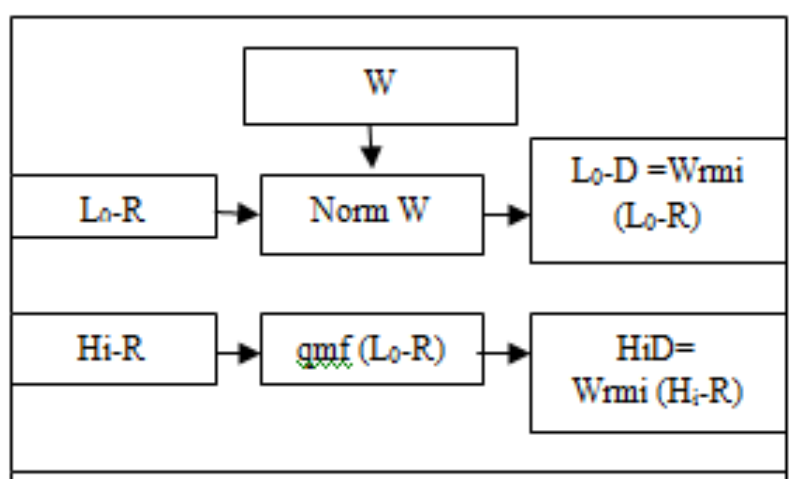

Fig.3( Four filters)

qmf : quadrature mirror filters

Wrmi: reconstruction mirror image of the filters

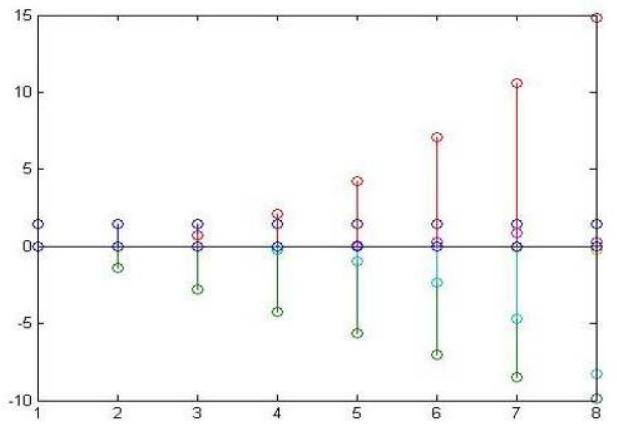

Fig.4 (Filters for the Laguerre wavelets)

The two filters reconstructed and linked by

$$
L_{0} R=\frac{W}{\|W\|}, H_{i} R_{k}=(-1)^{k-1} L_{0} R_{K+1-k}
$$

By using Mirror's theory, the two decomposion filters are obtained

$$
L_{O} D_{k}=L_{0} R_{K+1-k}, H_{i} R_{K+1-k} \text { for } \mathrm{k}=1,2, \ldots, \mathrm{K}
$$

Using DLWT equation (1) and Fig.1 in (4) and (5), the following figure is obtained.

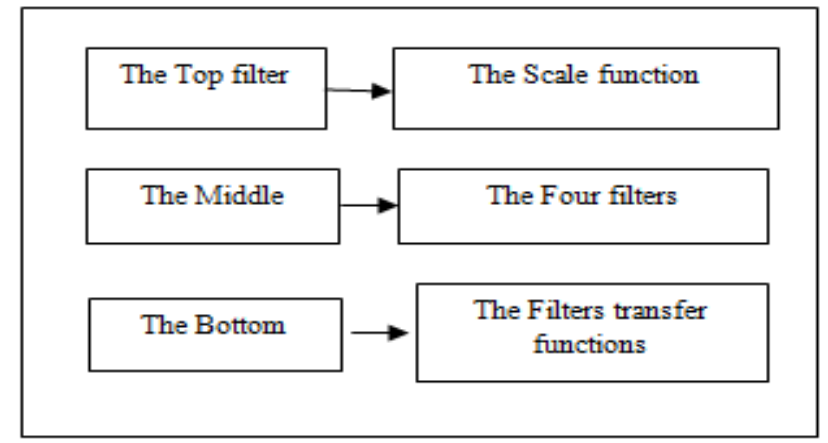

Fig.5 (Types of filters) 


\subsection{The Best Algorithm to Calculate the}

\section{Coefficients}

Algorithm 1: Processing signal by using DLWT One dimensional DLWT

Input :- Signal

_Output: processing signal

Step 1:- $A_{c o f}\left(C A_{1}\right), D_{c o f}\left(C D_{1}\right)$ are two sets

(the following filters)produced and obtained from transform $\mathrm{S}$ :

a- Low-Pass filter

b- High-Pass filter.

Between S\&F do Convolution see Fig.5

The convolution equation is

$$
[S * F]_{n}=\sum_{k} S_{n-k} F_{k}
$$

The decimation equation is

$$
Y=\operatorname{dec}(S) \bullet Y_{n}=S_{2 n}
$$

$\mathrm{LF}=2 \mathrm{~N}$, Let $\mathrm{N}=$ length $\mathrm{S}, \mathrm{G} \& \mathrm{~F}$ are signals with length $M+2 m-1$

The length of two coefficients $=$ floor $\frac{2 N}{2}+n$

Step 2:- The coefficients of the rounding coefficients are divided into two parts, replacing $s$ by $c A_{1}$ and producing $c A_{2}$ and $c D_{2}$, and so on see Fig.7

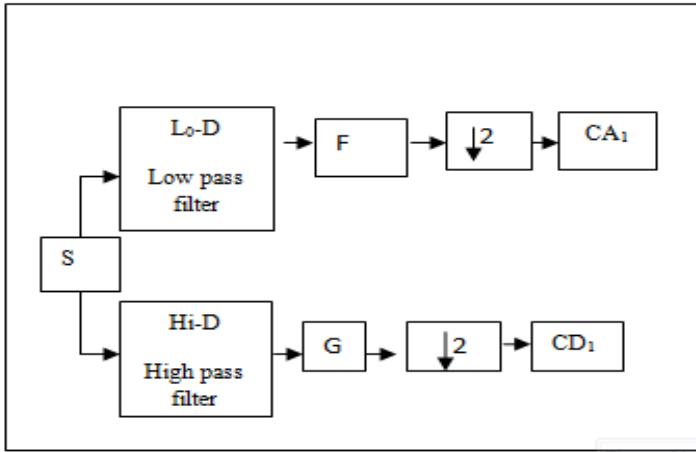

Fig.6 ( Demonstrates the decomposition of the signal in Level1 one dimension DLWT )

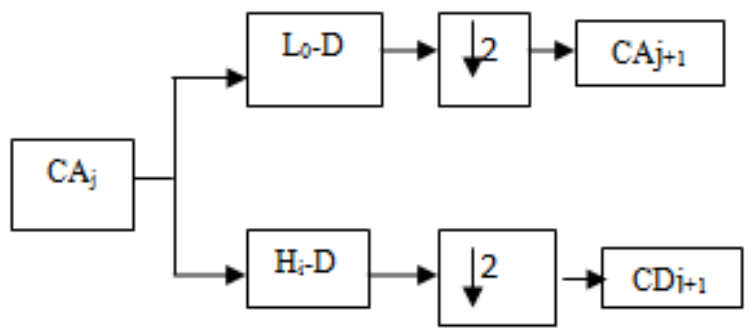

Fig.7 ( Representation of a DLWT decomposition step with initialization at $A^{0}=S$ )

\section{$\mathrm{X}$ : Convolution with filter $\mathrm{X}$}

$\downarrow 2$ Dawn Sample

From the above that the signal wave that dissociated from level $\mathrm{j}$ has the following structure: $\left\lfloor C A_{j}, C D_{j}, \ldots, C D_{1}\right\rfloor_{\text {The next }}$ stage will be the tree with nodes $\mathrm{j}=3$, which shows the signal structure

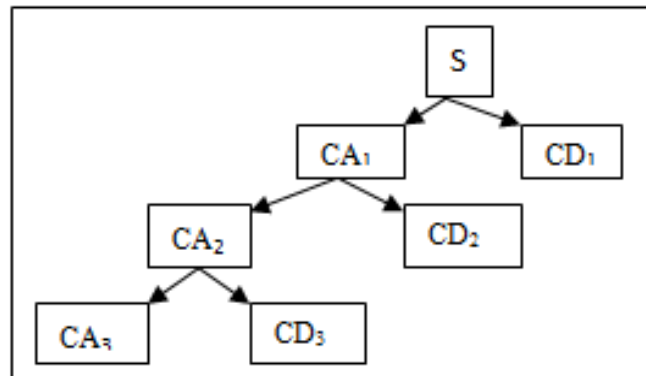

Fig.8 (One dimensional wavelets tree)

The following is a review of the functions that were built in a function approximation $f(t) \in L^{2}[0,1]$ may be expanded as

$$
f(t)=\sum_{n=1}^{\infty} \sum_{m=0}^{\infty} A_{n, m} \rho_{n, m}(t)
$$

$$
\text { where } \quad A_{n, m}=\left(f(t), \rho_{n, m}(t)\right)
$$

(10)

In equation (9), (., . ) denotes the inner product with weight function $w_{n}(t)$ on the Hilbert Space $[0,1)$ off the infinite series in the above equation is truncated, then equation (9) can be written as

$$
f(t)=\sum_{n=1}^{2^{k-1}} \sum_{m=0}^{M-1} A_{n, m} \rho_{n, m}(t)=A^{T} \rho_{n, m}
$$

Where $A$ and $\rho(t)$ are $2^{k-1} M \times 1$ matrices given by

$$
A=\left\lfloor A_{1,0}, A_{1,1}, \ldots, A_{1,(M-1)}, A_{2,0}, \ldots, A_{2^{k-1}, 0}, \ldots, A_{2^{k-1}, M-1}\right\rfloor^{T}
$$

$\rho=\left\lfloor\rho_{1,0}, \rho_{1,1}, \ldots, \rho_{1,(M-1)}, \rho_{2,0}, \ldots, \rho_{2^{k-1}, 0}, \ldots, \rho_{2^{k-1}, M-1}\right\rfloor^{T}$

Concerning orthonormal wavelet bases.

The good reason projection and doable scales:

Let $L_{V_{J}}$ and $L_{W_{J}}$ be the orthogonal projection operators, on $V_{j}$ and $W_{j}$ and for $f \in L^{2}$

If $A^{J}=L_{V_{J}} f$ and $D^{J}=L_{W_{J}} f$. The properties of spaces $\left\{V_{j}\right\}$ and $\left\{W_{j}\right\}$ imply where as

$A^{j-1}=A^{j}+D^{j}$ with $A^{j} \perp D^{j}$ 
Spaces $\left\{V_{j}\right\}$ are approach spaces in the following signification $A^{j}$ converges to $\mathrm{f}$ when $\mathrm{j}$ goes to 0 . In the same technique, spaces $\left\{W_{j}\right\}$ are detail spaces in the sense that $D^{j}$ are the difference between two alternate approximation i.e. $D^{J}=A^{J-1}-A^{J}$. Moreover, for fixed $\mathrm{J}$, the $D^{J}$ are the corrections to add to the roughest approximation $A^{J}$ in order to find f, since $f=A^{J}+\sum_{j=0}^{J} D^{j}$. In $\left(V_{j}+W_{j}\right)$ with $\left\{\vartheta_{j, m}\right\}_{m \in Z},\left\{\rho_{j, m}\right\}_{m \in Z}$ where $\vartheta, \rho$ are scaling function and wavelet associated with the Multi Resolution Analysis (MRA)

\section{DECOMPOSITION AND RECONSTRUCT OF DLWT}

\subsection{Decomposition one dimension DLWT}

In this section constructed algorithm1 taken from the Mallat algorithm. It is fast algorithm of decomposition-reconstruct. will dependent in formations from equations (9)-(13)

Let $\rho_{n, m}(t)=\left|a^{n}\right|^{-\frac{1}{2}} \rho\left(2^{-n} t-m\right), \forall n, m \in Z, a \neq 0$

From equation(14) and let $V_{n}, W_{n}$ are endowed with orthonormal bases scaling function from equation (2), when $\mathrm{n}=1,2, \ldots . \quad$ And $\quad \mathrm{m}=0 \vartheta_{n, 0}=\left\{\begin{array}{cc}1 & 0 \leq t \leq 1 \\ 0 & \text { otherwise }\end{array}\right.$ (15)

$\rho_{n, m}$ is wavelet function associated with the MRA for a function $\rho$, where $\rho_{n, m}=2^{-n} \rho\left(2^{-n} t-m\right)$.

Characterize the approach and detail coefficients by the following relations reasonable for all in $\mathrm{Z}$.

$$
\begin{aligned}
& A^{n}=\sum_{r \in z} a_{r}^{n} \vartheta_{n, r}, a_{r}^{n}=\left(A^{n}, \vartheta_{n, r}\right)_{L^{2}}=\left(f, \vartheta_{n, r}\right)_{L^{2}} \\
& D^{n}=\sum_{r \in z} d_{r}^{n} \rho_{n, r}, d_{r}^{n}=\left(D^{n}, \rho_{n, r}\right)_{L^{2}}=\left(f, \rho_{n, r}\right)_{L^{2}}
\end{aligned}
$$

The following algorithms will depend on the equations (14), (16) and (17).

1. Decomposition part:- calculate the co-ordinates $a^{n}$ and $d^{n}$ of approximation $A^{n}$ and detail $D^{n}$ using the co-ordinates $\boldsymbol{a}^{\boldsymbol{n}-\mathbf{1}}$ of the approximation $A^{n-1}$.

2. Reconstruction part:- the inverse operation is performed.

\section{Algorithm 2:}

Step 1. It starts with relations (14), (16) and (17)

$$
a_{m}^{n}=\left(A^{n-1}, \vartheta_{n, m}\right)_{L^{2}}, d_{n}^{m}=\left(A^{n-1}, \rho_{n, m}\right)_{L^{2}}
$$

From (14)

$$
\begin{aligned}
& \left(A^{n-1}, \vartheta_{n, m}\right)_{L^{2}}=\left(A^{n}+D^{n}, \vartheta_{n, m}\right)_{L^{2}} \\
& =\left(A^{n}, \vartheta_{n, m}\right)_{L^{2}}+\left(D^{n}, \vartheta_{n, m}\right)_{L^{2}}=a_{m}^{n}+0
\end{aligned}
$$

$\forall m \in Z, A^{n} \perp D^{n}$
Step 2. This step is same as step1, just replacing $\vartheta$ by $\rho$

$\left(A^{n-1}, \rho_{n, m}\right)_{L^{2}}=\left(A^{n}+D^{n}, \rho_{n, m}\right)_{L^{2}}$

$=\left(A^{n}, \rho_{n, m}\right)_{L^{2}}+\left(D^{n}, \rho_{n, m}\right)_{L^{2}}=0+d_{m}^{n}$

Step 3. Substituting $A^{n-1}=\sum_{r \in z} a_{r}^{n-1} \vartheta_{n, m}$ in (18)

$$
a_{m}^{n}=\sum_{r \in z} a_{r}^{n-1}\left(\vartheta_{n-1, r}, \vartheta_{n, m}\right)_{L^{2}}
$$

$d_{m}^{n}=\sum a_{r}^{n-1}\left(\vartheta_{n-1, r}, \rho_{n, m}\right)_{L^{2}}$

Step 4:- Calculate the scalar products in (19)

$$
\left\{\begin{array}{l}
\left(\vartheta_{n-1, r} \vartheta_{n, m}\right)_{L^{2}}= \\
\int 2^{-(n-1) / 2} \vartheta\left(2^{-(n-1)} x-r\right) 2^{-n / 2} \vartheta\left(2^{-n} x-m\right) d x \\
\left(\vartheta_{n-1}, \rho_{n, m}\right)_{L^{2}}= \\
\int 2^{-(n-1) / 2} \vartheta\left(2^{-(n-1)} x-r\right) 2^{-n / 2} \rho\left(2^{-n} x-m\right) d x
\end{array}\right.
$$

Step 5:- Changing the variable $\frac{y}{2}=2^{-n} x-m$, then $x=2^{n-1} y-2^{n} m$ obtain

$$
\begin{aligned}
& \left(\vartheta_{n-1, r}, \vartheta_{n, m}\right)_{L^{2}}=\sqrt{2} \int \vartheta(y-(r-2 m)) \frac{1}{2} \vartheta\left(\frac{y}{2}\right) d y \\
& \left(\vartheta_{n-1, r}, \rho_{n, m}\right)_{L^{2}}=\sqrt{2} \int \vartheta(y-(r-2 m)) \frac{1}{2} \rho\left(\frac{y}{2}\right) d y
\end{aligned}
$$

Step 6:- In this step verify the scaling equation by $\vartheta$ and the two scale equations defining the wavelet $\rho$ in equation (1)

$$
\begin{aligned}
& \left(\frac{1}{2}\right) \vartheta\left(\frac{y}{2}\right)=\sum_{n \in z} a_{n} \vartheta_{0, n}=\sum_{n \in z} a_{n} \vartheta(y-n) \\
& \left(\frac{1}{2}\right) \rho\left(\frac{y}{2}\right)=\sum_{n \in Z} b_{n} \vartheta_{0, n}=\sum_{n \in Z} b_{n} \vartheta(y-n) \\
& \left\{\vartheta_{n, m}\right\} \in V_{0}, \text { equation (20) }
\end{aligned}
$$$$
\left\{\begin{array}{l}
\left(\vartheta_{n-1, r}, \vartheta_{n, m}\right)_{L^{2}}=\sqrt{2} a_{r-2 m} \\
\left(\vartheta_{n-1, r}, \rho_{n, m}\right)_{L^{2}}=\sqrt{2} b_{r-2 m}
\end{array}\right.
$$

NOTE:- The results of the basic functions of the scales $n-1$ and $n$ do not depend on $\mathrm{n}$. Thus the same initial step of decomposition stratify to all the scales.

Stepe 7:-With respect to $a_{m}^{n}, d_{m}^{n}$ and from equations (19),(21)

$$
\left\{\begin{array}{l}
a_{m}^{n}=\sum_{r \in Z} \vartheta_{r}^{n-1} \sqrt{2} a_{r-2 m} \\
d_{m}^{n}=\sum_{r \in Z} \vartheta_{r}^{n-1} \sqrt{2} b_{r-2 m}
\end{array}\right.
$$

Stepe 8:- In this decomposition, phase is within the benefit of the previous equations that the following the two filters involved are initially determined. They were put in already for the special case of the finite filters. 
$\left\{\begin{aligned} L_{0} D & =\left\{\left[L_{0} D\right]_{k}\right\}_{k \in Z}\left(\because\left[L_{0} D\right]_{k}=\sqrt{2} a_{-k}\right) \\ H_{i} D & =\left\{\left[H_{i} D\right]_{k}\right\}_{k \in Z}\left(\because\left[H_{i} D\right]_{k}=\sqrt{2} d_{-k}\right)\end{aligned}\right.$

From (22)

$\left\{\begin{array}{l}a_{m}^{n}=\sum_{r \in Z} a_{r}^{n-1}\left[L_{0} D\right]_{2 m-r} \\ d_{m}^{n}=\sum_{r \in Z} a_{r}^{n-1}\left[H_{i} D\right]_{2 m-1}\end{array}\right.$

The operation of decimation

$y=\operatorname{dec}(x)$ is defined by $y_{k}-x_{2 k}$ for $k \in Z$. In filter

theory, decimation is often noted as $\downarrow 2$, and equation (23) is finally written as

$\left\{\begin{array}{l}a^{n}=\operatorname{dec}\left(a^{n-1}, L_{0} D\right) \\ d^{n}=\operatorname{dec}\left(a^{n-1}, H_{i} D\right)\end{array}\right.$

The result of above algorithm calculated is of approximation $A^{n}$ and detail $D^{n}$ and are therefore calculated simply using the coefficients of approximation $A^{n-1}$, a convolution (a filter) followed by decimation.

Result (24) is represented by the following figure

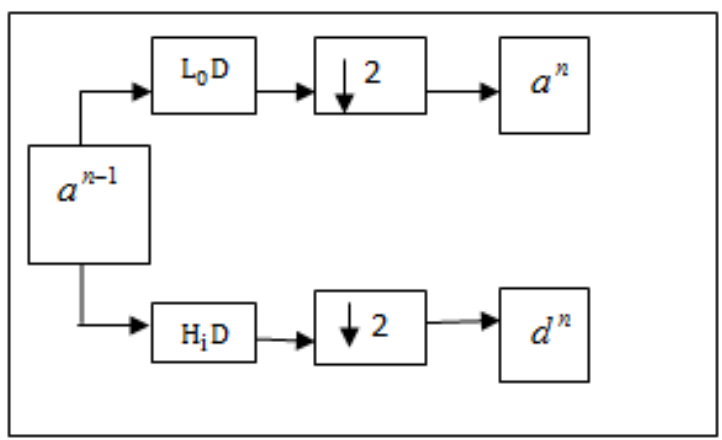

Fig.9 (representation of equation (20) and convolution by $\mathrm{F}$ and $\downarrow 2$ is decimation)

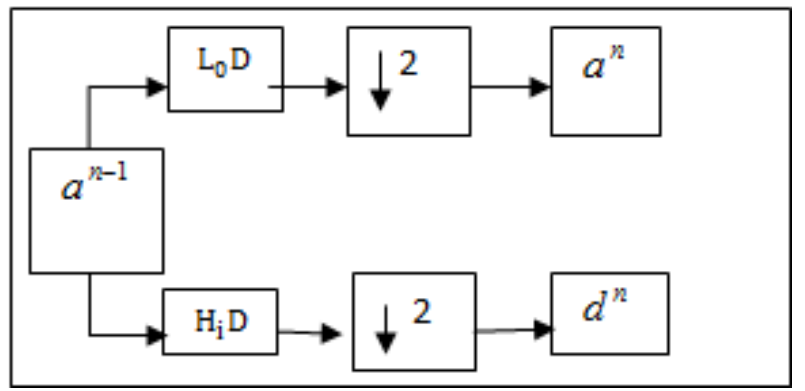

Fig.10 (compact representation of (20))

\subsection{The Reconstruction DLWT}

In this section the steps of the algorithm are counterproductive to calculate the co-ordinates of $A^{n-1}$ from $A^{n}$ and $D^{n}$.The following equality will be started, $\forall m \in Z$

$$
\begin{aligned}
& a_{m}^{n-1}=\left(A^{n-1}, \vartheta_{n-1, m}\right)_{L_{2}}=\left(A^{n}+D^{n}, \vartheta_{n-1, m}\right)_{L^{2}} \\
& =\left(A^{n}, \vartheta_{n-1, m}\right)_{L^{2}}+\left(D^{n}, \vartheta_{n-1, m}\right)_{L^{2}} \\
& \therefore a_{m}^{n-1}=\sum_{r \in Z} a_{r}^{n}\left(\vartheta, \vartheta_{n-1, m}\right)_{L^{2}}+\sum_{r \in z} d_{r}^{n}\left(\rho_{n, r}, \vartheta_{n-1, m}\right)_{L^{2}}
\end{aligned}
$$

From tep 7, the reconstruction two filters will be there.

$$
\left\{\begin{aligned}
L_{0} R & =\left\{\left[L_{0} R\right]_{k}\right\}_{k \in z} & & \left(\because\left[L_{0} R\right]_{k}=\sqrt{2} a_{k}\right) \\
H_{i} R & =\left\{\left[H_{i} R\right]_{k}\right\}_{k \in z} & & \left(\because\left[H_{i} R\right]_{k}=\sqrt{2} d_{k}\right)
\end{aligned}\right.
$$

NOTE: From the previous stage i.e. the decomposition stage can draw these two filters

$$
L_{0} R=\operatorname{inv}\left(L_{0} D\right) \text { and } H_{i} R=\operatorname{inv}\left(H_{i} D\right)
$$

Where $y=\operatorname{inv}(x), y(m)=x(-m), m \in Z$

Using relations (26) and (27),

$$
a_{m}^{n-1}=\sum_{r \in Z} a_{r}^{n}\left(L_{0} R\right)_{m-2 r}+\sum_{r \in Z} d_{r}^{n}\left(H_{i} R\right)_{m-2 r}
$$

Pressed the process of zeros admission defined by $y=\operatorname{ins}(x), y_{2 n}=x_{n}, y_{2 n-1}=0, k \in Z$ with in the filter theory admission is overwhelmingly denoted by $\uparrow 2$ and from relation (28),

$$
\begin{aligned}
& a_{m}^{n-1}=\sum\left[\operatorname{ins}\left(a^{n}\right)\right]\left(L_{0} R\right)_{m-r}+\sum\left[\operatorname{ins}\left(d^{n}\right)\right]\left(H_{i} R\right)_{m-r} \\
& a^{n}=\operatorname{ins}\left(a^{n}\right) * L_{0} R+\operatorname{ins}\left(d^{n}\right) * H_{i} R
\end{aligned}
$$

Finally from the above operations coefficients of approach $A^{n}$ and detail $D^{n}$ from the coefficients of approach $A^{n-1}$ by a convolution of linear filter represented in the following figures

\subsection{Decomposition of two dimension DLWT}

For images, an analogous algorithm is probable for twodimensional wavelets and scaling functions gained from onedimensional wavelets by sensorial product. From this type of two-dimensional DLWT, get a decomposition of approximation coefficients at level $\mathrm{j}$.

(a) The approximation at level $\mathrm{j}+1$

(b) The details in three orientations

- Horizontal.

- Vertical.

- Diagonal.

In general the following characterize the steps of MRA for images. Now the orthogonal wavelets, from the algorithms above but the decomposition filters and the reconstruction filters gained from two separate scaling functions related with MRA in dualism. The following algorithm will calculate approximation vector. 


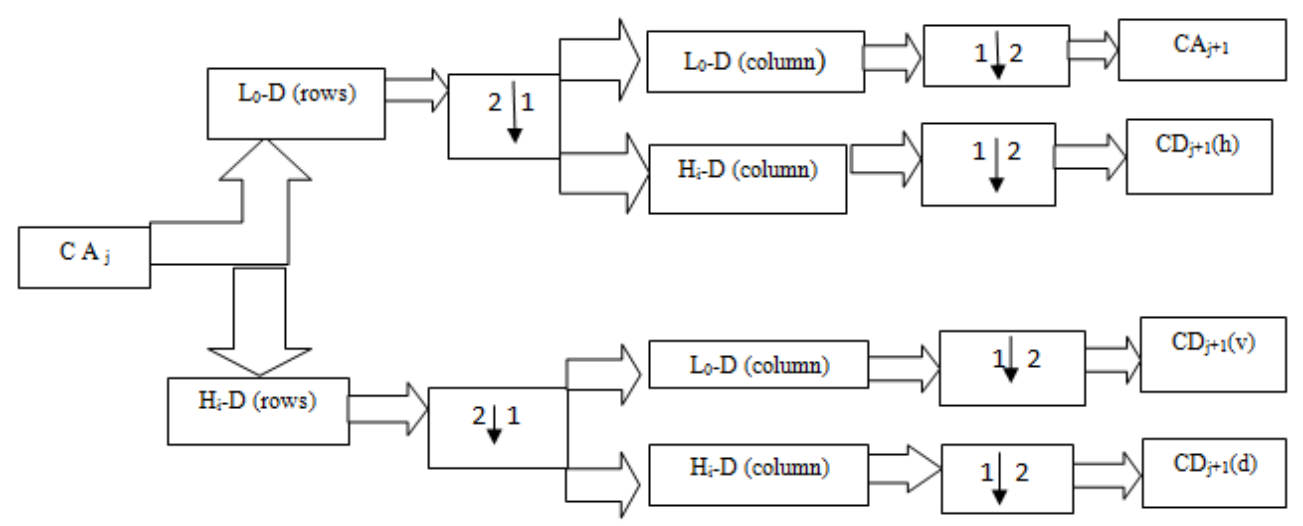

Fig.11 ( Decomposition of tow dimensional DLWT )

Algorithm3: This algorithm is to calculate approximation vector.

1. $\left(A_{m}(n)\right)_{m \in Z}$ be the coordinates of the vector $A_{n}$

$$
A_{n}=\sum A_{m}(n) \vartheta_{n, m}
$$

2. $A_{m}(n+1)$ be the coordinates of the vector $A_{n+1}$

$$
A_{n+1}=\sum_{m} A_{m}(n+1) \vartheta_{n+1, m}
$$

3. $A_{m}(n+1)$ Is calculated from following

$$
A_{m}(n+1)=\sum_{n} h_{n-2 m} \vartheta_{m}(n)
$$

4. This formula is like a convolution formula.

$\tilde{h}=h(-m), \quad F_{m}(n+1)=\sum \tilde{h}_{m-n} A_{m}(n)$

5. The sequence $F(n-1)$ is the filtered output of the sequence $A(n)$ by the filter $\tilde{h}$.we obtain.

$$
A_{m}(n+1)-F_{2 m}(n+1)
$$

It takes the even index values of $F$. This is down sampling.

The sequence $A(n+1)$ is the down sampled version of the Sequence $F(n+1)$. 
$2 \downarrow 1$ : Down sample column keep the even indexed columns.

$1 \downarrow 2$ : Down sample rows keep the even indexed rows.
$\mathrm{X}$ (columns) : Convolve with filter $\mathrm{X}$ the columns of the entry .

$\mathrm{CA}_{0}=\mathrm{S}$ for the decomposition initialization.

$\mathrm{X}$ (rows) : Convolve with filter $\mathrm{X}$ the rows of the entry .

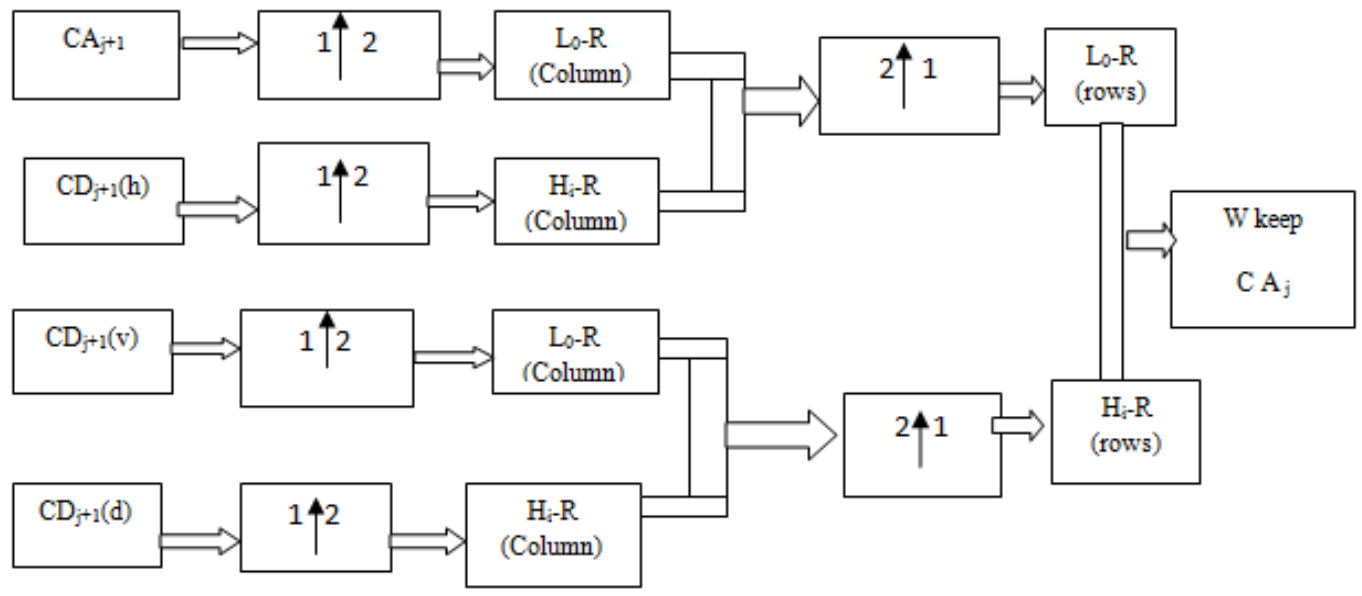

Fig.12 (Reconstruction of tow dimensional DLWT)

2 1: Up sample columns keep the odd indexed columns.

$1 \uparrow^{2}$ : Up sample rows keep the odd indexed rows.
$\mathrm{X}$ (rows): Convolve with filter $\mathrm{X}$ the rows of the entry.

$\mathrm{X}$ (Column): Convolve with filter $\mathrm{X}$ the columns of the entry.

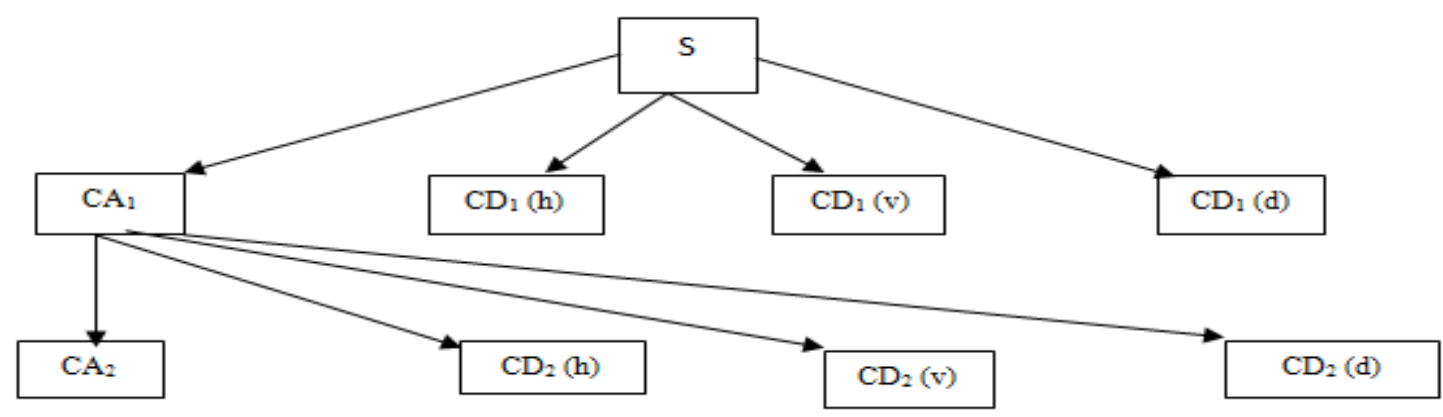

Fig.13 (Two dimensional wavelets tree)

By initial condition $A_{m}(0)=S(m)$,

By $S(m)$ is the signal value at time $m$.

The multi resolution situation and to a few of the properties

of the functions $\vartheta_{n, m}, \rho_{n, m}$. 


\section{Algorithm 4: Describe some of $\vartheta_{n, m}$ and $\rho_{n, m}$}

1. The family $\vartheta_{0, m}$ is formed of orthonormal functions. As a consequence for any $n$, the family $\vartheta_{0, m} m \in Z$ is orthonormal.

2. The double indexed family $\rho_{n, m} n, m \in Z$ is orthonormal.

3. For any $n$, the $\vartheta_{n, m}, m \in Z$ are orthogonal to $\rho_{n, m}, n \leq m \in Z$.

4. Between two alternate scales, we have an essential relation, called the twin-scale form.

$$
\vartheta_{1,0}=\sum_{n, m \in Z} h_{n} \vartheta_{n, m}, \vartheta_{m+1,0}=\sum h_{m} \vartheta_{n, m}
$$

5. This relation introduces the algorithm's $h$ filter $h_{k}=\sqrt{2 w_{k}}$

6. For more information check the following .

- The coordinate of $\vartheta_{n+1,0}$ on $\vartheta_{n, m}$ is $h_{m}$ and does not depend on $n$.

- $\quad$ The coordinate of $\vartheta_{n+1, k}$ on $\vartheta_{n, m}$ is equal to

$$
\left\langle\vartheta_{n+1, k}, \vartheta_{n, m}\right\rangle-h_{m-2 k}
$$

These relations supply the essential for the algorithm.

Thus

a. The filter $h$ is the low- pass filter .

b. The high-pass filter $g$

From a\&b we will obtained the two scales functions they are $\rho$ the following essential relation.

These relations supply the essential for the algorithm.

$$
\begin{gathered}
\rho_{1,0}=\sum_{k \in Z} g_{k} \vartheta_{0, k} \\
\rho_{j+1,0}=\sum_{k \in Z} g_{k} \vartheta_{j, k}
\end{gathered}
$$

These relations supply the essential for the algorithm.

Thus,

(a) The filter $h$ is the low-pass filter .

(b) The high-pass filter $g$

From (a) and (b) two scales functions and wavelet $\rho$ will be obtained. The following are the essential relations for the algorithm.

$$
\begin{aligned}
& \rho_{1,0}=\sum_{k \in Z} g_{k} \vartheta_{0, k} \\
& \rho_{j+1,0}=\sum_{k \in Z} g_{k} \vartheta_{j, k}
\end{aligned}
$$

After MRA steps, the reconstruction algorithm by building it, initial condition $A_{1}$ and $D_{1}$, are given by

$A_{0}=A_{1}+D_{1}$ The steps from $A_{n}=A_{n+1}+D_{n+1}$

and $a_{n}, \delta_{n}, a_{k}^{0}$ are given by

$A_{1}=\sum_{m} a_{m} \vartheta_{1, m}, D_{1}=\sum_{m} \delta_{m} \rho_{1, m}$,

$A_{0}=\sum_{m} a_{m}^{0} \vartheta_{0, m}$

Assume the $a_{k}^{0}$ coordinates as:

$$
\begin{aligned}
& a_{m}^{0}=\left\langle A_{0}, \vartheta_{0, m}\right\rangle=\left\langle A_{1}+D_{1}, \vartheta_{0, m}\right\rangle= \\
& \left\langle A_{1}, \vartheta_{0, m}\right\rangle+\left\langle D_{1}, \vartheta_{0, m}\right\rangle=\sum_{k} a_{k}\left\langle\vartheta_{1, k}, \vartheta_{0, k}\right\rangle+ \\
& \sum_{k} \delta_{k}\left\langle\rho_{1, k}, \vartheta_{0, m}\right\rangle=\sum_{k} a_{k} h_{m-2 k}+\sum_{k} \delta_{k} g_{m-2 k}
\end{aligned}
$$

In this step used the following relations.

$$
\begin{aligned}
& \sum_{k} a_{k} h_{m-2 k} \\
& \sum_{k} \delta_{k} g_{m-2 k}
\end{aligned}
$$

If $\mathrm{m}=0$, the above relations changes as

$$
\begin{gathered}
\tilde{a}_{2 k}=a_{k}+a_{2 k}+1+0 \\
\Rightarrow \sum_{k} a_{k} h_{-2 k}=\sum_{k} \delta_{k} h_{-k}
\end{gathered}
$$

$$
\begin{aligned}
& \text { By extension } \sum_{k} a_{k} h_{m-2 k}=\sum_{k} \delta_{k} h_{m-k} \\
& \text { since } a_{m}^{0}=\sum_{k} \tilde{a}_{k} h_{m-1}+\sum_{k} \tilde{\delta}_{k} g_{m-k}
\end{aligned}
$$

\section{Algorithm 5:-The reconstruction steps are:}

1- Replace the $\alpha$ and $\delta$ sequences by up sampled versions $\tilde{a}, \tilde{\delta}$ inserting zeros.

2- Filter by $h$ and $g$ respectively.

3- Sum the obtained sequences. 


\subsection{The Capabilities of DLWT on Dimensional}

Algorithm 6: Analysis signal one dimension

In put: original signal.

Out put: analysis signal one dimension

Step (1):-

Basic One- Dimensional Objects. Signal in original time $\mathrm{S}$ (Original signal)

$$
\begin{aligned}
& A_{m}, 0 \leq m \leq n \quad(\text { Approximate at level m) } \\
& \left.D_{m}, 1 \leq m \leq n \quad \text { (Detail at level } \mathrm{m}\right)
\end{aligned}
$$

$\operatorname{Step}(2):-$

Coefficients in scale-related time

$$
\begin{aligned}
& C A_{m}, 1 \leq m \leq n \quad(\text { A at level } \mathrm{m}) \\
& \left.C D_{m}, 1 \leq m \leq n \quad \text { (D at level } \mathrm{m}\right) \\
& {\left[C A_{n}, C D_{n}, \ldots, C D_{1}\right] \quad \text { W.D } n \geq 0}
\end{aligned}
$$

This Analysis are Decomposition Capabilities

Step (3):- synthesis are Reconstruction Capabilities.

DLWT

$$
S \rightarrow\left[C A_{1}, C D_{1}\right] \rightarrow\left[C A_{m}\right] \rightarrow\left[C A_{n+1}, C D_{n+1}\right]
$$

Wave dec $S \rightarrow\left[C A_{n}, C D_{n}, \ldots, C D_{1}\right]$

In this section will displayed basic One-Dimensional objects, in the following algorithm is displayed the resulting signals. Which file be used by using MATLAB programming.

\section{5- The Capabilities of DLWT Two-Dimensional}

Algorithm 7:- Basic Two-Dimensional Objects.
Step (1):- Image in original resolution
S (Original image) $A_{0}$ Approximation at level 0
$A_{m}, \quad 1 \leq m \leq n \rightarrow$ Approximation at level $\mathrm{m}$
$D_{m}, \quad 1 \leq m \leq n \rightarrow$ Detail at level m
Step (2):- Coefficients in scale-related resolution
$C A_{m}, 1 \leq m \leq n \rightarrow$ Approximation coefficients at
level m
$C D_{m}, 1 \leq m \leq n \rightarrow$ Detail coefficients at level m
$\left[C A_{n}, C D_{n}, \ldots, C D_{1}\right] \rightarrow$ Wavelet decomposition at
level $\mathrm{n}$

\section{APPLIED DISCRETE LAGUERRE WAVELET TRANSFORM}

After the image has been transformed in to the frequency or sequence domain, may want to modify the resulting spectrum. There are three types of filtering:-

Low pass filters tend to blur images. They pass low frequencies and attenuate or eliminate the high-frequency information. They are used for image compression or for hiding effects caused by noise. It imparts a softer effect to the image . Low-pass filtering is performed by multiplying the spectrum by a filter and then applying the inverse, can be used for edge enhancement because it passes only high-frequency information, corresponding to places where gray levels are changing rapidly (edges in images are characterized by rapidly changing gray levels) transform to obtain the filtered image.

\subsection{The band pass and band reject filters}

The band pass and band reject filters are specified by two cut off frequencies, a low cut off and a high cut off shown. A special form of these filters is called a notch filter because it only notches out or passes specific frequencies. These three types of filters are typically used in image restoration, enhancement and compression.

The wavelet transform is really a family of transforms that satisfy specific conditions. The wavelet transform can be described as a transform that has basis functions that are shifted and expanded versions of themselves. The wavelet transform contains not just frequency information but special information as well. One of the most common models for a wavelet transform uses the Fourier transform and high pass and low pass filters. The use of the wavelet transform is increasingly popular for image compression

Numerous filters can be used to implement the Wavelet Transform. One of the commonly used is the DLWT. It is separable. So they can be used to implement a wavelet transform by first convolving them with rows and then with columns. The DLWT basis vectors are simple: Use equation (1) 
for $t \in[0,1]$, and using equations (4) and (5) moreover applying above algorithms,

LOW PASS: $\frac{1}{\sqrt{2}}\left[\begin{array}{ll}1 & 1\end{array}\right]$
HIGH PASS: $\frac{1}{\sqrt{2}}\left[\begin{array}{ll}1 & -1\end{array}\right]$

From the above filters started to applied compressed image,

The following algorithm will illustrate this.

\section{Algorithm (8): Laguerre Wavelet's Filters}

Step (1):- If $m=0$ and $n=1,2$ then

$$
C_{n, 0}=\left\{\begin{array}{cc}
\frac{1}{\sqrt{2}}, & 0 \leq t \leq 1 \\
0, & \text { otherwise }
\end{array}\right.
$$

The first row

$$
f_{1}=\frac{1}{\sqrt{2}}\left[\begin{array}{ll}
1 & 1
\end{array}\right]
$$

Then Low_pass filter $\left[\begin{array}{ll}1 & 1\end{array}\right]$

Step ( 2) :- If $m=0,1$ and $n=1,2$ then

$$
C_{1,1}=\frac{1}{\sqrt{2}}, \quad C_{2,1}=-\frac{1}{\sqrt{2}}
$$

The second row

$$
\begin{gathered}
f_{2}=\frac{1}{\sqrt{2}}\left[\begin{array}{ll}
1 & -1
\end{array}\right] \\
C_{n, 1}= \begin{cases}\frac{1}{\sqrt{2}}, & 0 \leq t \leq 0.5 \\
\frac{-1}{\sqrt{2}}, & \text { otherwise }\end{cases}
\end{gathered}
$$

Then High pass filter $\left[\begin{array}{ll}1 & -1\end{array}\right]$

Step (3):- Programming and storage $F_{2 \times 2}=\frac{1}{\sqrt{2}}\left[\begin{array}{cc}1 & 1 \\ 1 & -1\end{array}\right]$

End
The following examples are processing image by using the coefficients of DLWT where $n=1$ and $m=0,1$. using visual basic program. But this way is not practical because it does not contain the programs ready compared to the programs Matlab, which use more practical and faster in the processed of images . 


\section{Examples 1}
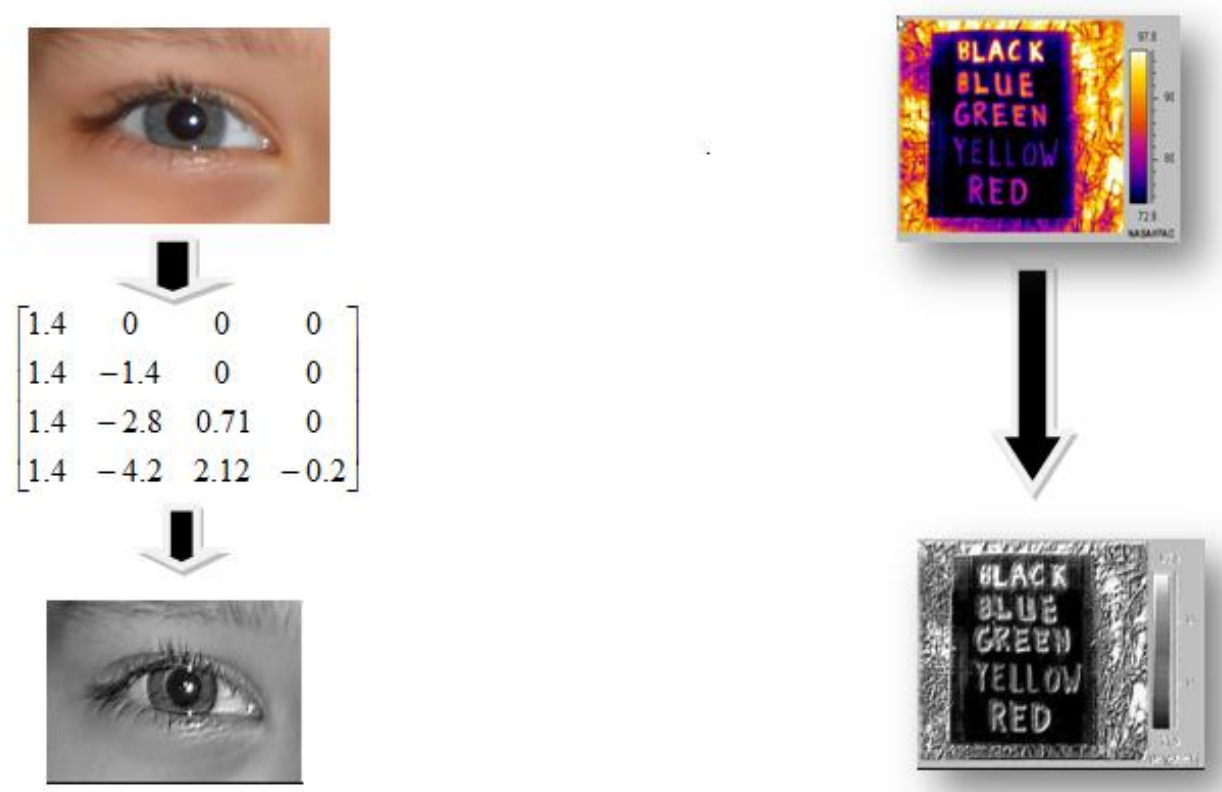

\section{Example 2}
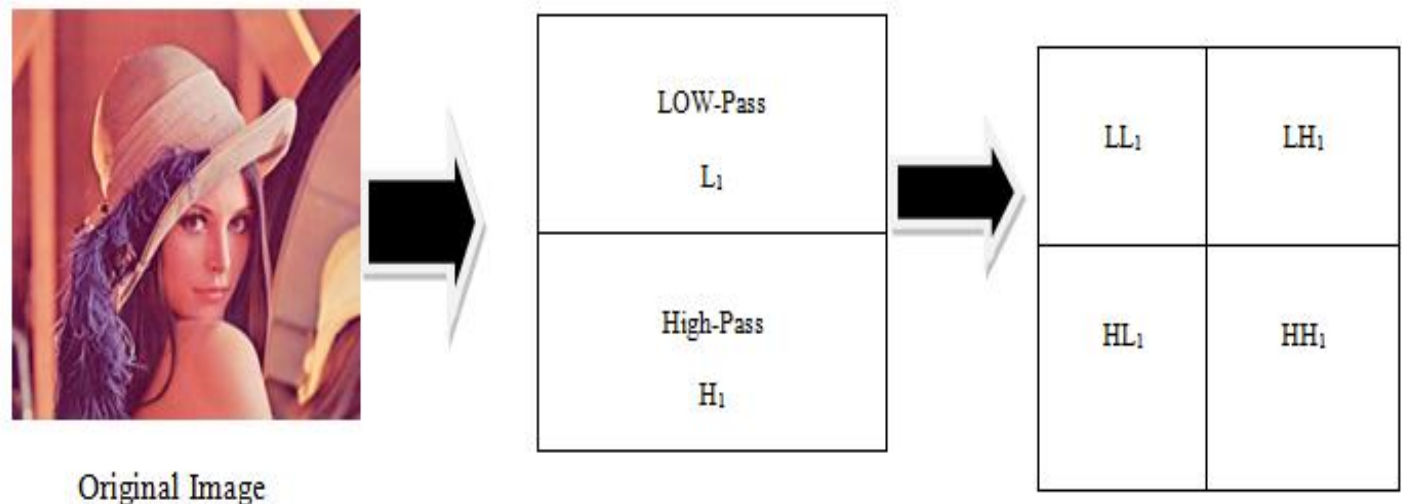

Original Image 

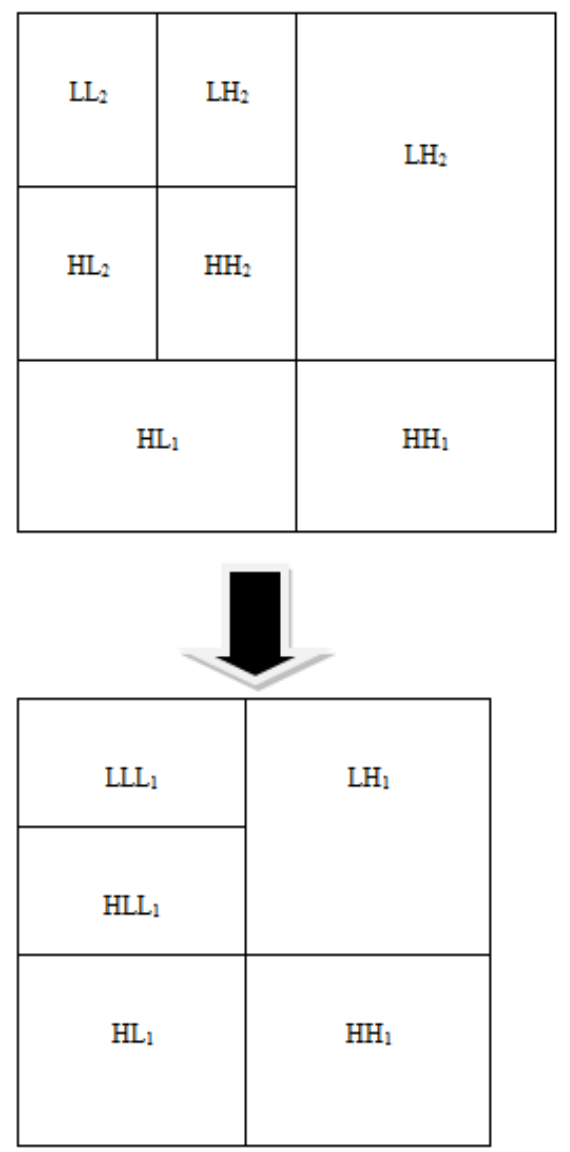

\section{CONCLUSION}

In thise paper authors used new form of wavelet that symbolizes Discrete Laguerre Wavelets Transform (DLWT) and its filters matrix for image processing without loss in its features with some algorithms, used fast algorithm to construct, how to calculate main coefficients and decision tree. Two examples are illustrated the images are compressed with the use of Matlab program after load DLWT is used to compress images and reduce noise. This work can be developed using an extended matrix of filters to reach more accurate results where the error ratio is zero in future.

\section{REFERENCES}

[1] A. M. Raid, and W. M. Khedr, ' JPEG Image Compression Using Discrete Cosine Transform- A Survey' International Journal of Computer Sciences\& Engineering Survey (IJCSES) VOL (5), NO (2), PP(39-47), (2014).
[2] Arpita C. Raut, Dr. R. R Sedamkar, ' Adaptive Super Spatial prediction Approach For Lossless Image Compression' International Journal of Engineering research and Applications, VOL(4), PP(175-180), (2014).

[3] B. Niteesh, V. Ravi Kumar, 'Image Compression Using Adoptive Haar Wavelet Based Tetorlet Transform' International Journal of Innovation Research in Computer and Communication Engineering (IJIRCCE), VOL(4), PP(6522-6528), (2016).

[4] Bahman Zafarifar, 'Micro - Cod able Discrete Wavelet Transform', Computer Engineering Laboratory Faculty of Information Technology and Systems Delft University of Technology The Netherlands, (2002).

[5] Gullanar M.Hadi, 'Medical Image Compression Using DCT and DWT Techniques ', ADVANCES IN IMAGE AND VIDEO PROCESSING VOL(2),PP(26-35), (2014).

[6] G.P.NASON, 'Wavelet Shrinkage Using Cross-Validation' Journal of the Royal Statistical Society Series B. VOL(58) PP(463-479), (1996).*

[7] Juanli HU, Jiabin Deng, 'Image Compression Based on Improved FFT Algorithm'Journal of Networks, VOL(6), NO(7), PP(1041-1048), (2011).

[8] Jan. E. Odegard \& Ivan. W. Jelesnick, 'Introduction To Wavelets and Wavelet Transform' BOOK.

[9] Khushpreet Kaur, Sheenam Malhtra, 'Image Compression Using Haar Wavelet Transform and Discrete Cosine Transform', International Journal of Computer Applications VOL(125), PP(28-31), (2015).

[10] Mridul Kumar, Gunjan Mathur, 'Image Compression Using DFT Through Fast Fourier Transform Technique', International Journal of Emerging Trends And Technology In Computer Science (IJETTCS), VOL(1), PP(129-133), (2012).

[11] M.Moza mmel Hoque \& Amina Khatun, 'Image Compression Using Discrete Wavelet Transform', International Journal of Computer Science Issues (IJCSI), VOL(9), NO(1), PP(327-330), (2012).

[12] Prab Hjot Kour , 'Image Processing Using Discrete Wavelet Transform' IPASJ, International Journal of Electronics \& Communication (IIJEC), VOL(3), PP(53-59), 2015.

[13] V. Ashok, T. Bala kumaran, 'The Fast Wavelet Transform for Signal And Image Processing', International Journal of Computer Science and Information Security, (IJCSIS), VOL(7), NO(1), PP(126-130), (2010). 